\title{
An innovative integral field unit upgrade with 3D-printed micro-lenses for the RHEA at Subaru
}

Theodoros Anagnos ${ }^{\mathrm{a}, \mathrm{b}, \mathrm{c}}$, Pascal Maier ${ }^{\mathrm{d}, \mathrm{e}}$, Philipp Hottinger ${ }^{\mathrm{c}}$, Christopher H. Betters ${ }^{\mathrm{i}}$, Tobias

Feger $^{\mathrm{a}, \mathrm{j}}$, Sergio G. Leon-Savali ${ }^{\mathrm{i}}$, Itandehui Gris-Sánchez ${ }^{\mathrm{g}, \mathrm{k}}$, Stephanos Yerolatsitis ${ }^{\mathrm{g}}$, Julien Lozi $^{\mathrm{h}}$, Tim A. Birks ${ }^{\mathrm{g}}$, Sebastian Vievard ${ }^{\mathrm{h}}$, Nemanja Jovanovic ${ }^{\mathrm{l}}$, Adam D. Rains ${ }^{\mathrm{o}}$, Michael J.

Ireland $^{\mathrm{o}}$, Robert J. Harris ${ }^{\mathrm{c}, \mathrm{p}}$, Blaise C. Kuo Tiong ${ }^{\mathrm{a}, \mathrm{b}}$, Olivier Guyon ${ }^{\mathrm{h}}$, Barnaby Norris ${ }^{\mathrm{i}}$, Sebastiaan Y. Haffert ${ }^{\mathrm{m}, \mathrm{n}}$, Matthias Blaicher ${ }^{\mathrm{d}, \mathrm{e}}$, Yilin $\mathrm{Xu}^{\mathrm{d}, \mathrm{e}}$, Moritz Straub ${ }^{\mathrm{q}}$, Jörg-Uwe Pott ${ }^{\mathrm{p}}$, Oliver Sawodny ${ }^{\mathrm{q}}$, Philip L. Neureuther ${ }^{\mathrm{q}}$, David W. Coutts ${ }^{\mathrm{a}, \mathrm{b}}$, Christian Schwab ${ }^{\mathrm{a}, \mathrm{b}}$, Christian $\operatorname{Koos}^{\mathrm{d}, \mathrm{e}, \mathrm{f}}$, and Andreas Quirrenbach ${ }^{\mathrm{c}}$

aDepartment of Physics and Astronomy, Macquarie University, NSW 2109, Australia

${ }^{b} \mathrm{MQ}$ Photonics Research Centre, Department of Physics and Astronomy, Macquarie University, NSW 2109, Australia

${ }^{\mathrm{c}}$ Landessternwarte, Zentrum für Astronomie der Universität Heidelberg, Königstuhl 12, 69117 Heidelberg, Germany

dInstitute of Microstructure Technology (IMT), Karlsruhe Institute of Technology (KIT),

Hermann-von-Helmholtz-Platz 1, 76344 Eggenstein-Leopoldshafen, Germany

e Institute of Photonics and Quantum Electronics (IPQ), Karlsruhe Institute of Technology (KIT), Engesserstr. 5, 76131 Karlsruhe

${ }^{\mathrm{f}}$ Vanguard Photonics GmbH, Hermann-von-Helmholtz-Platz 1,76344

Eggenstein-Leopoldshafen, 76227 Karlsruhe

'Department of Physics, University of Bath, Claverton Down, Bath, BA2 7AY, UK

${ }^{\text {h} N a t i o n a l ~ I n s t i t u t e s ~ o f ~ N a t u r a l ~ S c i e n c e s, ~ S u b a r u ~ T e l e s c o p e, ~ N a t i o n a l ~ A s t r o n o m i c a l ~}$

Observatory of Japan, Hilo, Hawaii, United States

${ }^{i}$ University of Sydney, Sydney Institute for Astronomy, Institute for Photonics and Optical

Science, School of Physics, Camperdown, Australia

${ }^{j}$ Redback Systems Pty Ltd, Sydney, Australia

kITEAM Research Institute, Universitat Politècnica de València, Camino de Vera, 46022

Valencia, Spain

${ }^{1}$ California Institute of Technology, 1200 E. California Blvd., Pasadena CA, 91125, USA

mLeiden Observatory, Leiden University, PO Box 9513, Niels Bohrweg 2, 2300 RA Leiden, The Netherlands

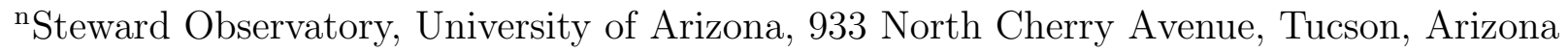

${ }^{\circ}$ Research School of Astronomy and Astrophysics, Australian National University, Canberra, ACT 2611, Australia

pMax-Planck-Institute for Astronomy, Königstuhl 17, 69117, Heidelberg, Germany

qInstitute for System Dynamics, University of Stuttgart, Waldburgstr. 19, 70563 Stuttgart, Germany

\begin{abstract}
In the new era of Extremely Large Telescopes (ELTs) currently under construction, challenging requirements drive spectrograph designs towards techniques that efficiently use a facility's light collection power. Operating in
\end{abstract}

Further author information: (Send correspondence to Th.A.)

E-mail: anagnos.theodoros@gmail.com 
the single-mode (SM) regime, close to the diffraction limit, reduces the footprint of the instrument compared to a conventional high-resolving power spectrograph. The custom built injection fiber system with 3D-printed microlenses on top of it for the replicable high-resolution exoplanet and asteroseismology spectrograph (RHEA) at Subaru in combination with extreme adaptive optics of SCExAO, proved its high efficiency in a lab environment, manifesting up to $\sim 77 \%$ of the theoretical predicted performance.

Keywords: astrophotonics, spectroscopy, micro-lenslets, SCExAO, radial velocity, optical fibers, fiber injection, diffraction-limited spectrograph, integral field unit

\section{INTRODUCTION}

A wealth of crucial information can be collected through astronomical spectroscopy, such as chemical composition, motion parameters as well as the indirect discovery of celestial bodies in orbit around other stars. ${ }^{1}$

Conventional spectrograph designs began to make use of fibers half a century ago ${ }^{2,3}$ in order to enable more efficient observations, as it became possible to locate the instrument off the telescope. Soon after, fiber based integral field unit (IFU) systems were developed ${ }^{4}$ that allowed flexibility in arranging spectra on a given detector space. Early on, multi-mode fibers (MMFs) were used for the IFU, which had high throughput for seeing-limited starlight (e.g. Ref. 5,6). Afterwards, new designs of IFU systems emerged taking advantage of single-mode fibers (SMFs) (e.g. Ref. 7,8).

Using SMFs and operating in the diffraction limit reduces the footprint of the instrument, however major limitations apply in coupling efficiency under seeing-limited conditions. By making use of the extreme adaptive optics (ExAO) systems installed in state-of-the-art telescopes, the coupling efficiency gets significantly better (e.g. Ref. 9,10).

While high-spatial resolution spectroscopy is achieved by using SM-IFUs, giving access to many new science capabilities, the coupling losses are high due to the low fill fraction of SMFs and the requirement for sub- $\mu \mathrm{m}$ precision in alignment.

In this study, we present an upgrade of the IFU system on the RHEA at Subaru. ${ }^{11,12}$ This custom IFU makes use of a multi-core fiber (MCF) with 19 SM cores, with 3D-printed micro-lenses on top of the cores manufactured by the two-photon polymerization lithography technique. ${ }^{13,14}$ This custom injection system significantly increases the free-space coupling of starlight into the fiber cores while allowing more tolerance for misalignment errors in targeting. The IFU system is optimized using Zemax optical software for instantaneous angular sky areas of 11 and 18 milli-arcseconds (mas) per lenslet. The system also offers a relatively high coupling efficiency and fill factor due to the 3D-printed micro-lens array (MLA).

In Section 2 we present the core design and parameters, complemented by the detailed experimental design description for the characterization of its performance. In Section 3 the laboratory results are presented. We draw conclusions in Section 4 and detail our future plans in Section 5.

\section{METHODS}

To increase the efficiency of light coupling from the 8-m Subaru telescope into the IFU feeding the RHEA, the following components are necessary: the Subaru Coronagraphic Extreme Adaptive Optics (SCExAO) system, the IFU itself with the 3D printed MLAs, the MCF and the spectrograph adapted to the output of the MCF. Below, these components are presented in more detail.

\subsection{Initial simulations}

In order to simulate the intensity distribution in the entrance of the IFU system, all the optical components of the SCExAO were taken into account. First of all, the output beam of the 8-m Subaru telescope undergoes adaptive optics (AO) correction in the AO188 unit and is then routed to the visual bench of SCExAO. The beam intensity distribution at this stage can be described as an Airy pattern.

For an efficient coupling of the Airy disk into the SM cores of the fiber, a special injection unit is necessary. This unit is specially designed to match the specifications of the MCF. The MCF has a $6.1 \mu \mathrm{m}$ mode-field 


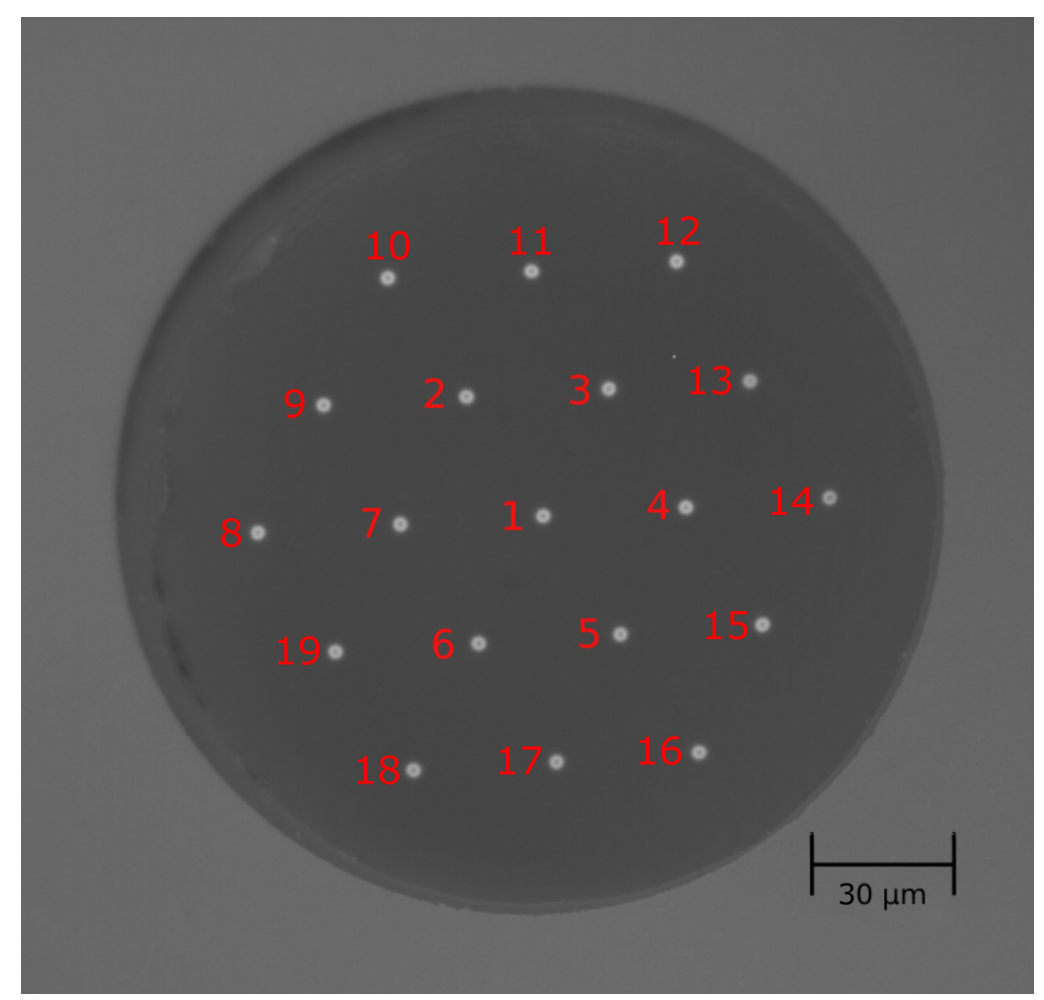

Figure 1. MCF end face after polishing and packaging into an FC/PC connector. The cores are positioned in a hexagonal formation with a pitch of $30 \mu \mathrm{m}$. Each of the cores has a $6.1 \mu \mathrm{m} \mathrm{MFD}$ at $600 \mathrm{~nm}\left(1 / \mathrm{e}^{2}\right)$. A HeNe laser source was used to back illuminate the fiber cores, which are labeled with red numbers for referencing.

diameter (MFD) at $650 \mathrm{~nm}\left(1 / \mathrm{e}^{2}\right)$ where the SM cut-off limit is at $\sim 600 \mathrm{~nm}$ (Figure 1 shows a microscope image of the MCF end face). The cores have a pitch of $30 \mu \mathrm{m}$ in hexagonal formation, which leads to a ratio of 4.9:1, giving enough separation to eliminate the cross-coupling between cores. The 3D printed structure of the MLAs is applied directly on top of the fiber end face using the two-photon lithography technique described below. This custom IFU will be installed into RHEA to maximize the system potential. The structure of the MLAs was optimized for a platescale of 11 and 18 mas on the sky per lenslet given that the diffraction limit of the Subaru telescope with the SCExAO at $650 \mathrm{~nm}$ is 17 mas.

\subsection{The visual SCExAO infrastructure}

The SCExAO is located in the near-infrared (NIR) Nasmyth focus of the $8 \mathrm{~m}$ Subaru Telescope. A complete and far more detailed description of both the visual and NIR paths of SCExAO is provided in Ref. 15. A brief overview starts with the starlight entering the Subaru Telescope and a 30-40\% Strehl correction on the point spread function (PSF) for the H-band accomplished by AO188. ${ }^{16-18}$ The starlight then enters the SCExAO NIR bench, where the wavefront is further corrected for higher-order aberrations caused by the atmosphere. In the next step, a dichroic filter separates the light beam in two paths: the visible $(<900 \mathrm{~nm})$ and the NIR $(>900 \mathrm{~nm})$ channel. Finally, the light beam is focused down with optical lenses onto the 3D printed MCF surface (see Figure 2).

\subsection{Throughput simulations}

For our simulations, an Airy disk was used an an input to the IFU system, taking into account the Subaru telescope profile and the key components of SCExAO. ${ }^{9,15}$

The simulation of the the Airy profile was performed by using the physical-optics propagation (POP) module of Zemax. ${ }^{19}$ This Airy disk output feeds a performance optimizer that selects for the best MLA structure to be $3 \mathrm{D}$ printed on top of the MCF. POP operands were used for varying MLA geometries that affect the Airy 


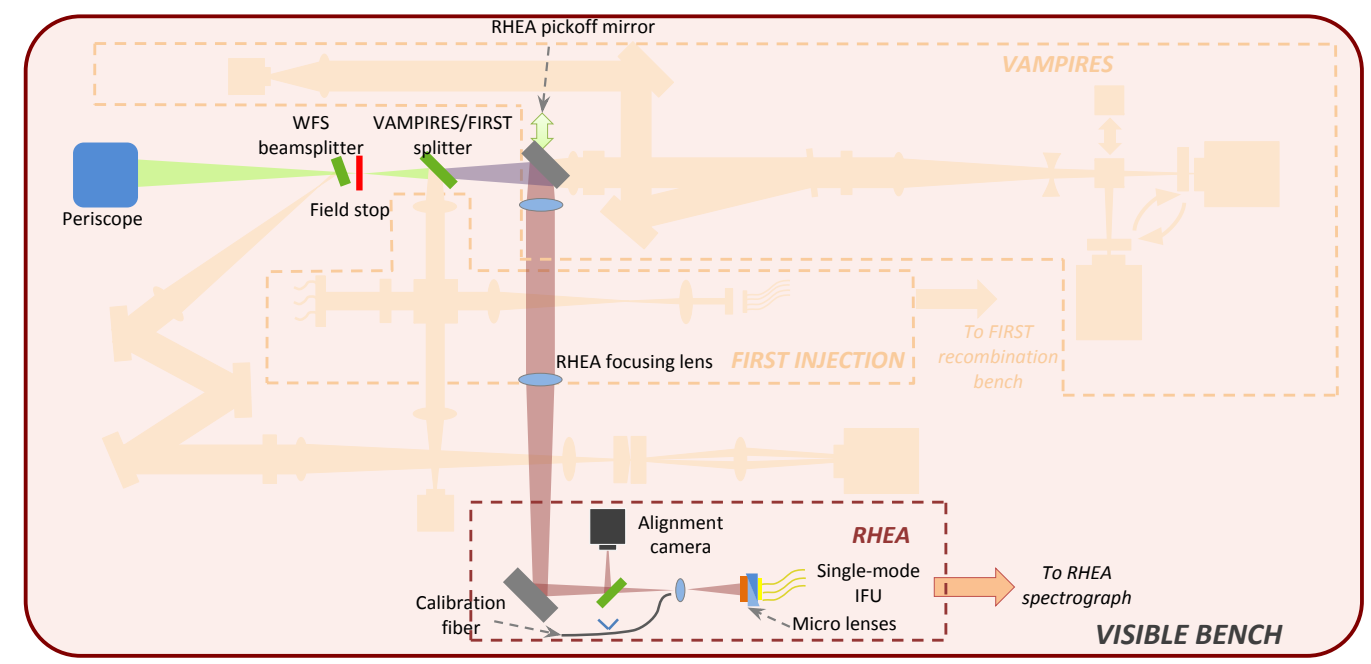

Figure 2. The visual bench of the SCExAO. The IFU injection is located in the bottom center of the illustration. The input beam from AO188 to the periscope to the IFU is represented with green and brown color.

beam coupling into the MCF cores. Several geometrical shapes were tested for the MLA structure in order to increase the coupling efficiency into the cores. Finally, a spherical surface was selected, as the alternatives provided negligible improvement in performance. A spherical surface MLA of $272 \mu \mathrm{m}$ in height and $115 \mu \mathrm{m}$ in radius of curvature achieved the highest coupling efficiency for the wavelength range of $600-800 \mathrm{~nm}$, resulting in a throughput of $50 \%$ for the 18 mas platescale for the central lens and a throughput of $21.9 \%$ for the platescale of 11 mas for the central lens. To achieve a fill factor of $\sim 100 \%$ in between the cores of the MCF, the micro-lenses were merged together forming a hexagonal effective aperture. The roughness of the MLA structure is expected to be better than $\lambda / 16-\lambda / 21$ at the working wavelength, as a surface roughness of $37 \mathrm{~nm}$ is achieved using the $3 \mathrm{D}$ printing technique. ${ }^{20}$

\subsection{Micro-lens array fabrication}

The MLA was printed to the cleaved facet of the MCF as a single model block using the commercially available negative-tone photoresist IP-Dip ${ }^{21}$ and an in-house built two-photon lithography machine. This system is equipped with a $780 \mathrm{~nm}$ femtosecond laser $^{22}$ and a 40x Zeiss objective lens with numerical aperture (NA) $=1.4$. A custom control software was developed in-house to guarantee optimum shape-fidelity of the printed MLA and allow for high-precision alignment with respect to the fiber cores of the MCF.

As a first step, the MCF was manually glued to an FC-PC connector and subsequently polished to achieve a flat fiber end-facet accessible for the lithography machine for printing. Thereafter, the fiber is back-illuminated by coupling in the light of a red light-emitting diode (LED) to accommodate machine vision for the detection of the 19 cores of the MCF. After the detection procedure, the individual lenses of the MLA are aligned with respect to the detected core positions of the $\mathrm{MCF}$, thereby taking into account variations of the core positions and pitch. All individually positioned lenses are then merged into a single 3D-model of the MLA to prevent unnecessary double-illumination in the overlap regions during the printing process. The structure is further automatically adapted to compensate for any tilt of the fiber end-facet. For the purpose of reducing the required printing time, the MLA is divided into two parts: the first block of the model up to just below the lens surfaces was written with a distance between subsequent layers, i.e., slicing distance, of $600 \mathrm{~nm}$. For optimal printing quality, the remaining second model block comprising the 19 lens surfaces of the MLA was written with a slicing distance of $100 \mathrm{~nm}$. The writing distance between subsequent lines, i.e., hatching distance, was set to $100 \mathrm{~nm}$ throughout the full model. The fabricated structure was afterwards developed in propylene-glycol-methyl-etheracetate (PGMEA), flushed with isopropanol, and subsequently blow dried. In the next stage, scanning electron microscopy (SEM) and vertically-scanned white-light interferometry (VSI) images of the structure were acquired to check the quality of the manufacturing process (see Figure 3). 


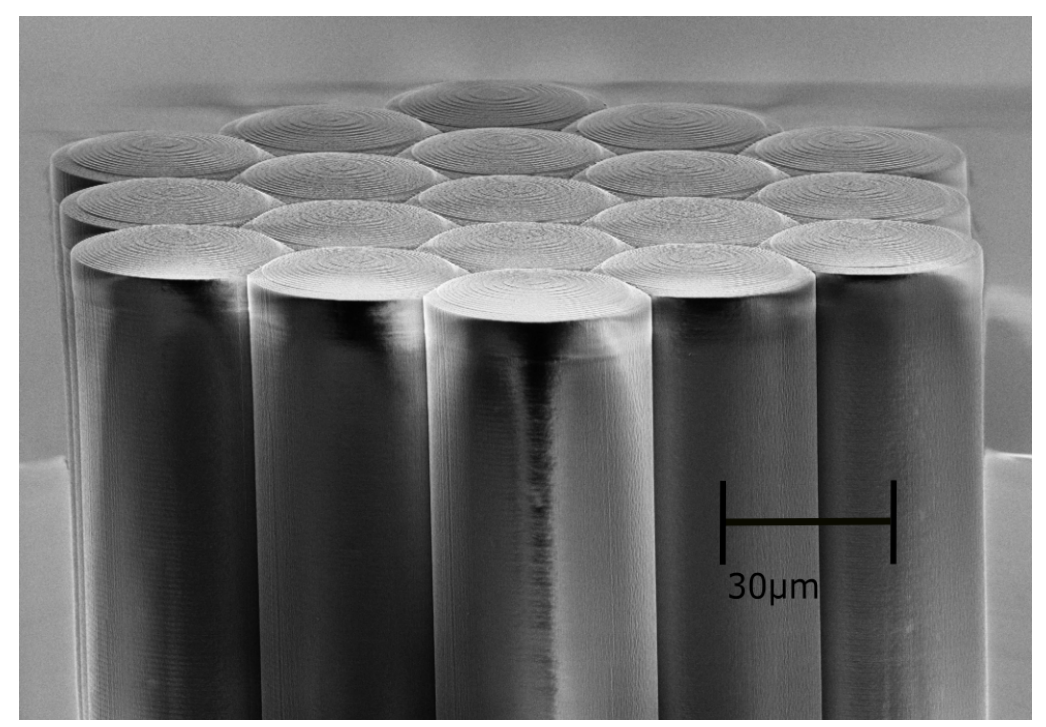

Figure 3. The top structure of the 3D printed MLA on top of the MCF end face acquired using the SEM technique.

\subsection{Laboratory throughput measurement setup}

For measuring the throughput performance of the custom 3D-printed MCF, a set of opto-mechanical parts was constructed as an addition to the Königstuhl Observatory Opto-mechatronics Laboratory (KOOL) test-bed. The throughput setup is presented in Figure 4. The HeNe laser light $(632 \mathrm{~nm})$ passes through a 50:50 non-polarizing beamsplitter (BS) L1 (Thorlabs CM1-BS014) and is collimated using an achromat lens L2 (Thorlabs AC127-025B-ML). Later on, the beam is split using another 50:50 non-polarizing BS L4 (Thorlabs CM1-BS014). One beam is focused down using an $100 \mathrm{~mm}$ achromat (AC254-100-B-ML) to the CMOS detector (Thorlabs DCC1545M), and the other is routed through a flip mirror L6 to an achromat L7 (AC254-060-B-ML or AC254-100-B-ML depending on the platescale) and focused down to the 3D-printed MCF that is mounted onto a 4-axis mount (Thorlabs MBT401D). After that, the fiber exit is re-imaged using a combination of achromats L8 and L9 (AC127-019-B-ML and AC127-050-B-ML) to the CMOS detector (Thorlabs DCC1545M). To calculate the total throughput of the MCF including the the coupling losses, a power meter was used (Thorlabs S120C) in order to perform the measurements and calibrate absolute flux through the re-imaging system.

\section{RESULTS}

\subsection{Throughput efficiency results}

To asses the performance of the custom IFU system before installation into RHEA, laboratory tests were performed as described in section 2.5. The outcome of these tests are presented here.

As mentioned in section 2.5 the throughput measurements were monochromatic using a HeNe laser at $632 \mathrm{~nm}$. After a series of optical elements in the KOOL test-bed, the beam was focused down to the 3D-printed MCF. Data frames with exposure times of a fraction of a second were collected with both achromats L7 (AC254-060B-ML or AC254-100-B-ML), using the setup described above. The setup was able to sample the near-field of the MCF exit and filter the light between the adjacent cores of the fiber. Averaged dark data frames were recorded as well, and subtracted from the data for further processing.

To characterize the absolute performance of the IFU, two separate experiments were conducted; in the first, the total coupling efficiency of the light into each of the 19 cores was determined after the alignment of the cores on-axis with the injected beam. In the second experiment, the misalignment tolerances of the injected beam were measured by translating the injected beam by steps of $5 \mu \mathrm{m}$ in respect to the central core of the MCF. This was more representative of realistic on-sky conditions where the star would be moving due to atmospheric perturbations. 


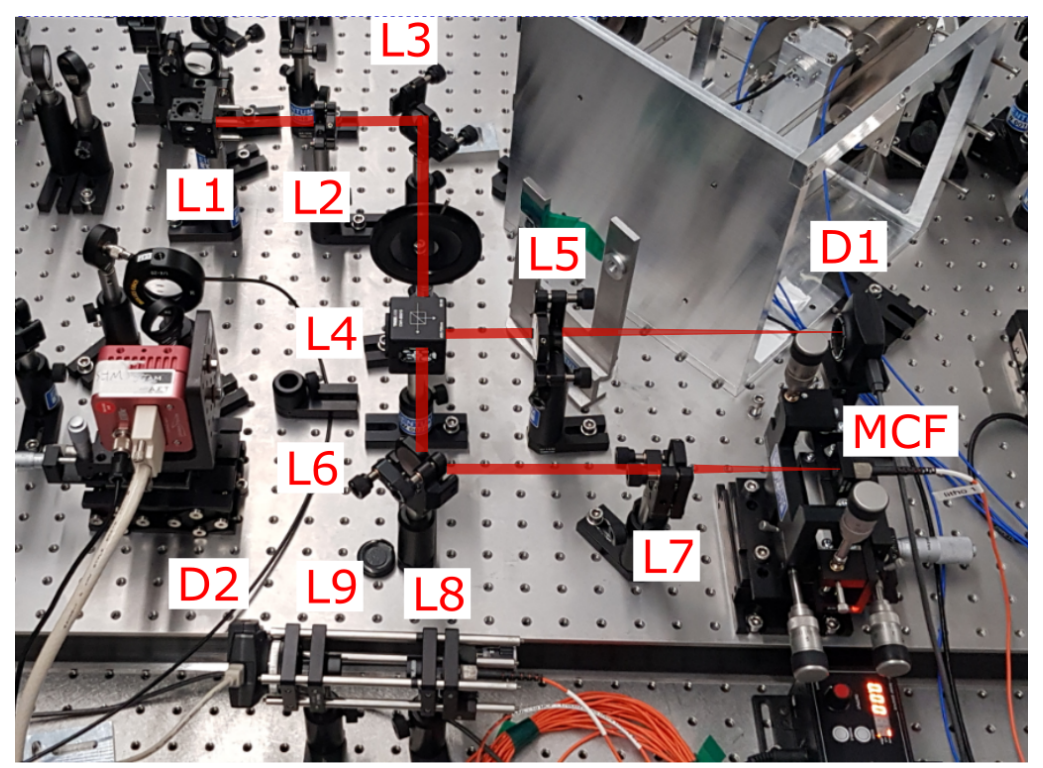

Figure 4. The throughput experimental setup for measuring the efficiency of the custom IFU. A power meter is used to calibrate the throughput. A set of achromat lenses (L2-L5-L7) is used for collimation and focusing of the beam, beamsplitter (BS) and CMOS detector (D1, D2) for imaging the near-field output of the MCF (L8, L9).

The results of the first experiment for the 18 mas platescale are shown in Figure 6. The average coupling efficiency was $21.45 \pm 3 \%$ with a maximum of $30.89 \pm 3 \%$ for the core \#11. The maximum measured throughput corresponds to $77.41 \%$ of the simulated value $(40 \%)$ calculated with Zemax. For the 11 mas platescale, the coupling efficiency of the central core was $16.9 \pm 3 \%$ ( $77.2 \%$ of the simulated value). The total throughput from all of the cores summed was calculated, representative of an unresolved target, and was measured to be $41.5 \pm 2 \%$. The residual coupling losses are associated with the imperfectly polished fiber, Fresnel reflections $(\sim 4 \%)$, and mode-field mismatch at the focus.

In Figure 7 the results from the second experiment are presented. This illustration shows the coupling tolerances as a function of off-axis target injection for the case of 18 mas. This demonstrates the potential of 3D-printed MLA technology for misaligned targets, showing fairly good coupling efficiency even for an off-axis injection of $\sim 10 \mu \mathrm{m}$, retaining $\sim 40 \%$ of the maximum throughput.

\section{CONCLUSIONS}

In this work we presented a novel IFU system upgrade for RHEA at the Subaru telescope. This IFU is composed of a custom MCF with 3D printed micro-lenses on top of the cores to increase the coupling efficiency for off and on-axis targets from SCExAO at the Subaru $8 \mathrm{~m}$ telescope. The IFU system is optimized using the Zemax POP module for an on-sky angular dimension of 11 and 18 mas using an Airy profile beam produced by the visual arm of SCExAO.

The custom MCF is composed of 19 cores in the same cladding with a core-to-core spacing of $30 \mu \mathrm{m}$ and a $6.1 \mu \mathrm{m} \mathrm{MFD} \mathrm{at} 650 \mathrm{~nm}\left(1 / \mathrm{e}^{2}\right)$ which leads to a negligible cross-coupling between the cores. The cores are positioned in a hexagonal formation and their cut-off SM limit is above $600 \mathrm{~nm}$.

The structure of the MLA was manufactured with two-photon lithography and 3D-printed on top of the cores of the MCF, significantly enhancing the throughput of light into the fiber cores from levels of few percent to a maximum of $30.89 \pm 3 \%$ for on-axis targets for a platescale of 18 mas. Furthermore, the custom MLA improved the off-axis light losses even for a $10 \mu \mathrm{m}$ lateral injection. The throughput performance across all the MLA as a representative of a single unresolved target was $41.5 \pm 2 \%$. The laboratory results correspond to $\sim 77 \%$ of the simulated results. The difference in throughput performance from simulated results are likely associated with the imperfectly polished MCF, Fresnel reflections ( $4 \%)$ and mode-field mismatch at the focus. These lab results were performed at the KOOL test-bed with a HeNe laser source $(632 \mathrm{~nm})$. 

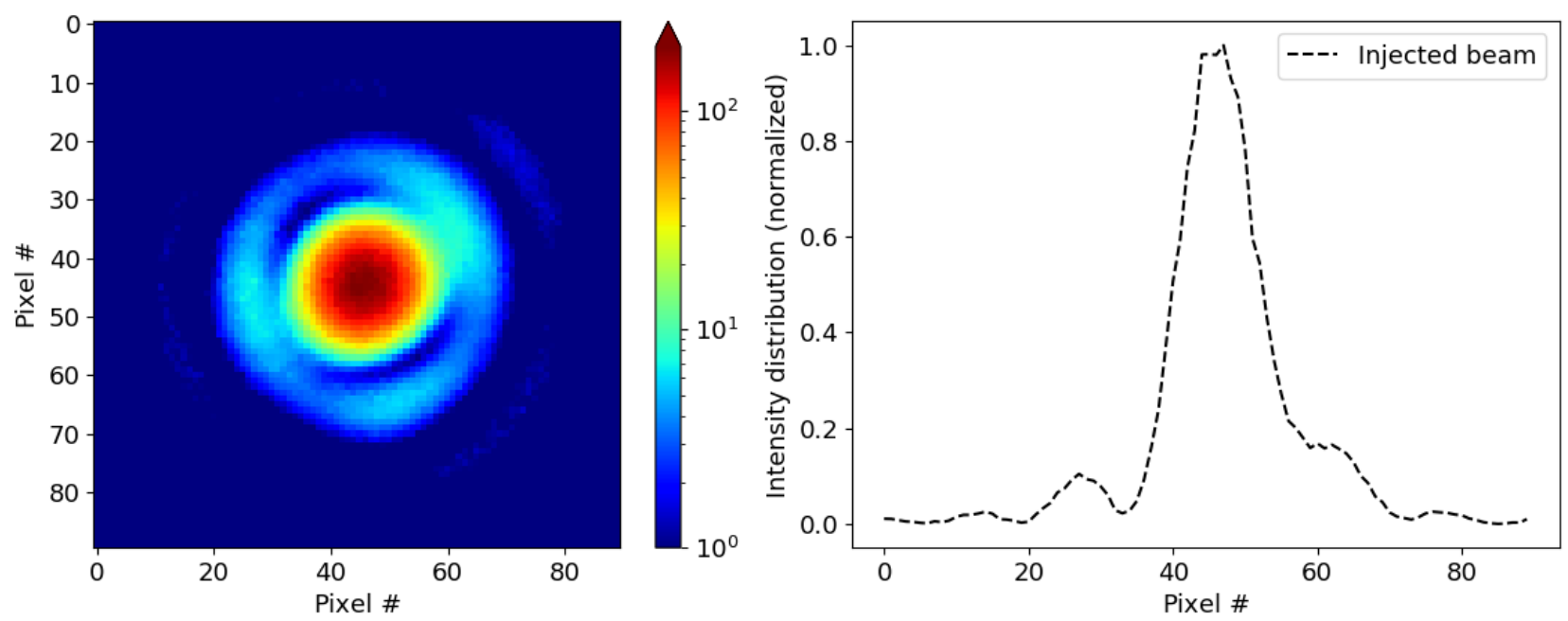

Figure 5. Left panel: 2D image of the injected beam in logarithmic color scale for better clarity, as measured in the laboratory. Right panel: Intensity profile of the injected point spread function normalized to its maximum, from the $2 \mathrm{D}$ image data.

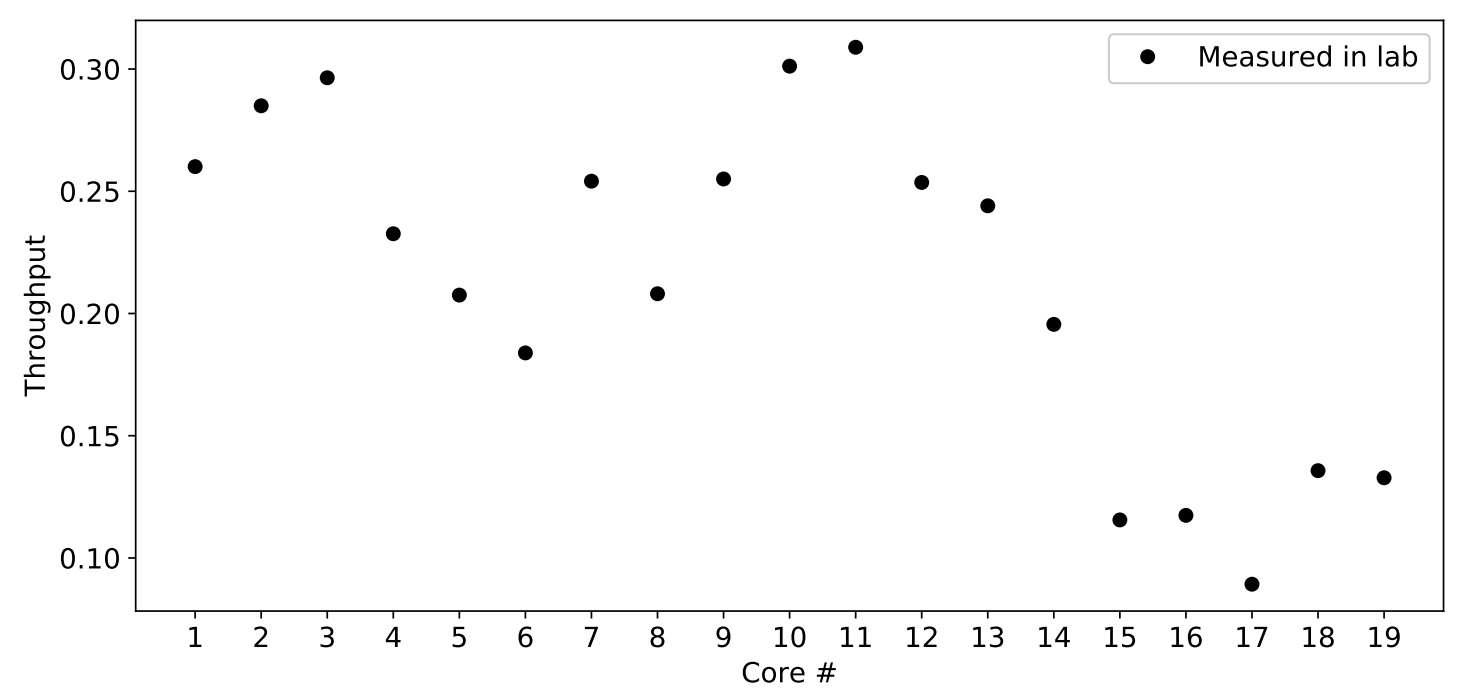

Figure 6. Coupling efficiency for all of the cores of the MCF (see Figure 1 for the numbering of the cores). This is shown for the 18 mas platescale. 


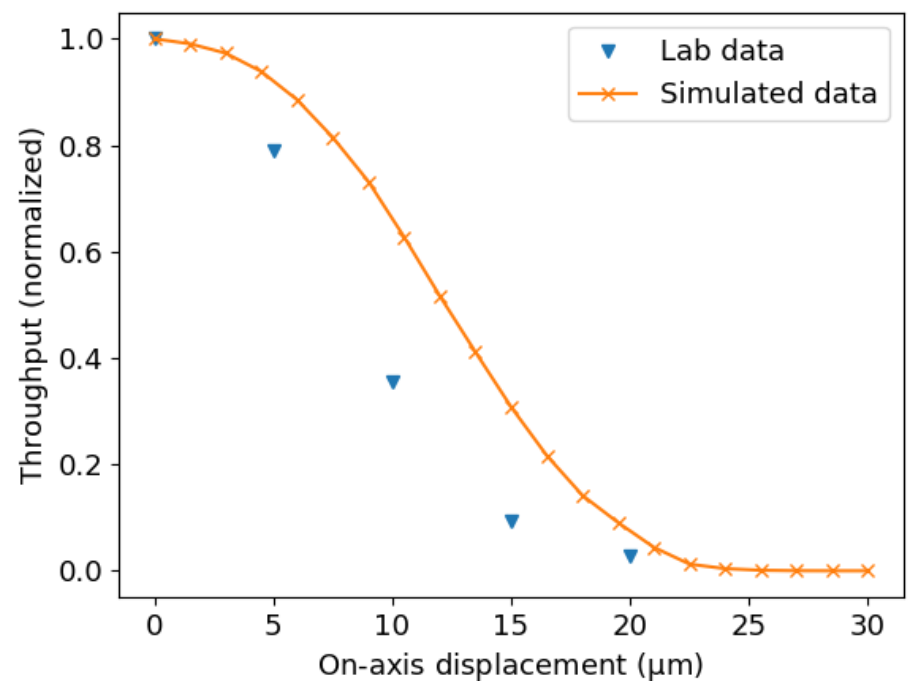

Figure 7. Coupling efficiency of the central core of the MCF as a function of off-axis target, compared with the simulated data from Zemax. Results are normalized to the maximum coupling efficiency including the errors (smaller than the data points).

\section{FURTHER WORK}

Plans for further work include a separate 3D-printed MCF, optimized for only the 18 mas platescale with Zemax simulations. Both IFU systems will be tested on the KOOL test bed including the effect of atmospheric turbulence using the AO system of the KOOL infrastructure.

Future work will be to integrate the fibers into the RHEA and perform on-sky tests on a variety of targets (resolved, un-resolved stars, confirmed exoplanets, spectroscopic standard stars and double star systems) in order to probe its scientific potential.

\section{ACKNOWLEDGMENTS}

T.A. is a fellow of the International Max Planck Research School for Astronomy and Cosmic Physics at the University of Heidelberg (IMPRS-HD) and is supported by the Cotutelle International Macquarie University Research Excellence Scholarship. P.M., M.B., Y.X. and C.K. are supported by Bundesministerium für Bildung und Forschung (BMBF), joint project PRIMA (13N14630), the Helmholtz International Research School for Teratronics (HIRST), Deutsche Forschungsgemeinschaft (DFG, German Research Foundation) under Germany's Excellence Strategy via the Excellence Cluster 3D Matter Made to Order (EXC2082/1-390761711). R. J. H. and P.H. are supported by the Deutsche Forschungsgemeinschaft (DFG) through project 326946494, 'Novel Astronomical Instrumentation through photonic Reformatting'. T.B. \& S.Y. are supported from the European Union's Horizon 2020 grant 730890, and from the UK Science and Technology Facilities Council grant ST/N000544/1. S.Y.H. is supported by the NASA Hubble Fellowship grant \#HST-HF2-51436.001-A awarded by the Space Telescope Science Institute, which is operated by the Association of Universities for Research in Astronomy, Incorporated, under NASA contract NAS5-26555. The development of SCExAO was supported by the JSPS (Grant-in-Aid for Research \#23340051, \#26220704 \#23103002), the Astrobiology Center (ABC) of the National Institutes of Natural Sciences, Japan, the Mt Cuba Foundation and the directors contingency fund at Subaru Telescope, and the OptoFab node of the Australian National Fabrication Facility. The authors wish to recognize and acknowledge the very significant cultural role and reverence that the summit of Maunakea has always had within the indigenous Hawaiian community. We are most fortunate to have the opportunity to conduct observations from this mountain. This research made use of Astropy, a community-developed core Python package for Astronomy, ${ }^{23,24}$ Numpy $^{25}$ and Matplotlib. ${ }^{26}$ Furthermore, this publication makes use of data generated at the 
Königstuhl Observatory Opto-mechatronics Laboratory (KOOL) which is run at the Max-Planck-Institute for Astronomy (MPIA, PI Jörg-Uwe Pott, jpott@mpia.de) in Heidelberg, Germany. KOOL is a joint project of the MPIA, the Landessternwarte Königstuhl (LSW, Univ. Heidelberg, Co-I Philipp Hottinger), and the Institute for System Dynamics (ISYS, Univ. Stuttgart, Co-I Prof. Oliver Sawodny). KOOL is partly supported by the German Federal Ministry of Education and Research (BMBF) via individual project grants.

\section{REFERENCES}

[1] P. Massey and M. M. Hanson, "Astronomical Spectroscopy," Planets, Stars and Stellar Systems. Volume 2: Astronomical Techniques, Software and Data, 35 (2013).

[2] E. N. Hubbard, J. R. P. Angel, and M. S. Gresham, "Operation of a long fused silica fiber as a link between telescope and spectrograph.," ApJ 229, 1074-1078 (May 1979).

[3] J. R. Powell, "Application of optical fibres to astronomical instrumentation.," Proc. SPIE 445, 77-84 (1984).

[4] J. Allington-Smith, "Basic principles of integral field spectroscopy," New A Rev 50, 244-251 (June 2006).

[5] J. Ge, J. R. P. Angel, and J. C. Shelton, "Optical spectroscopy with a near-single-mode fiber-feed and adaptive optics," Proc. SPIE 3355, 253 - 263 (1998).

[6] S. M. Croom, J. S. Lawrence, J. Bland-Hawthorn, et al., "The Sydney-AAO Multi-object Integral field spectrograph," MNRAS 421, 872-893 (Mar. 2012).

[7] S. G. Leon-Saval, C. H. Betters, and J. Bland -Hawthorn, "The Photonic TIGER: a multicore fiber-fed spectrograph," Proc. SPIE 8450, 84501K (2012).

[8] M. Tamura, H. Suto, J. Nishikawa, et al., "Infrared Doppler instrument for the Subaru Telescope (IRD)," Proc. SPIE 8446, $84461 \mathrm{~T}$ (2012).

[9] N. Jovanovic, C. Schwab, O. Guyon, et al., "Efficient injection from large telescopes into single-mode fibres: Enabling the era of ultra-precision astronomy," A\&SA 604, A122 (Aug. 2017).

[10] O. Guyon, R. Belikov, E. Bendek, et al., "Wavefront Sensing and Control R\&amp;D on the SCExAO Testbed," American Astronomical Society 51, 280.06 (Jan. 2020).

[11] T. Feger, C. Bacigalupo, T. R. Bedding, et al., "RHEA: the ultra-compact replicable high-resolution exoplanet and Asteroseismology spectrograph," Proc. SPIE 9147, 91477I (Aug 2014).

[12] A. D. Rains, M. J. Ireland, N. Jovanovic, et al., "Precision single mode fibre integral field spectroscopy with the RHEA spectrograph," Proc. SPIE 9908, 990876 (Aug 2016).

[13] P.-I. Dietrich, R. J. Harris, M. Blaicher, et al., "Printed freeform lens arrays on multi-core fibers for highly efficient coupling in astrophotonic systems," Optics Express 25, 18288 (jul 2017).

[14] P. Hottinger, R. J. Harris, P.-I. Dietrich, et al., "Micro-lens array as tip-tilt sensor for single-mode fiber coupling," in [SPIE Astronomical Telescopes + Instrumentation], International Society for Optics and Photonics (2018).

[15] N. Jovanovic, F. Martinache, O. Guyon, et al., "The Subaru Coronagraphic Extreme Adaptive Optics System: Enabling High-Contrast Imaging on Solar-System Scales," PASP 127, 890 (Sept. 2015).

[16] Y. Hayano, H. Takami, O. Guyon, et al., "Current status of the laser guide star adaptive optics system for Subaru Telescope," Proc. SPIE 7015, 701510 (2008).

[17] Y. Hayano, H. Takami, S. Oya, et al., "Commissioning status of Subaru laser guide star adaptive optics system," Proc. SPIE 7736, 77360N (2010).

[18] Y. Minowa, Y. Hayano, S. Oya, et al., "Performance of Subaru adaptive optics system AO188," Proc. SPIE 7736, 77363N (2010).

[19] Zemax, "Opticstudio - zemax," (2016).

[20] P.-I. Dietrich, M. Blaicher, I. Reuter, et al., "In situ 3d nanoprinting of free-form coupling elements for hybrid photonic integration," Nature Photonics 12(4), 241-247 (2018).

[21] Nanoscribe GmbH, "Ip photoresists," (2018).

[22] Menlo Systems GmbH, "C-fiber 780 femtosecond erbium laser," (2020).

[23] Astropy Collaboration, T. P. Robitaille, E. J. Tollerud, et al., "Astropy: A community Python package for astronomy," A\&A 558, A33 (Oct. 2013). 
[24] A. M. Price-Whelan, B. M. Sipőcz, H. M. Günther, et al., "The Astropy Project: Building an Open-science Project and Status of the v2.0 Core Package," AJ 156, 123 (Sept. 2018).

[25] S. van der Walt, S. C. Colbert, and G. Varoquaux, "The NumPy Array: A Structure for Efficient Numerical Computation," Computing in Science and Engineering 13, 22-30 (Mar. 2011).

[26] J. D. Hunter, "Matplotlib: A 2D Graphics Environment," Computing in Science and Engineering 9, 90-95 (May 2007). 